\title{
Albumin infusion for the critically ill - is it beneficial and, if so, why and how?
}

\author{
Undurti N Das ${ }^{1,2}$
}

\section{Introduction}

Human albumin is used for volume expansion and resuscitation and to correct hypoalbuminemia [1]. Hypoalbuminemia is frequent in sepsis and is a strong predictor of mortality and morbidity [2]. Fluid volume expansion and resuscitation of the critically ill with albumin has been recommended by the UK National Institute for Health and Care Excellence and the Surviving Sepsis Campaign (grade $2 \mathrm{C}$ ), although the evidence for these recommendations is not strong [1,3-8] and use of albumin to correct or improve hypoalbuminemia remains controversial $[7,8]$ despite the fact that albumin is safe to use for the critically ill [8].

Albumin mobilizes polyunsaturated fatty acids (PUFAs) from the liver and other tissues, and thus enhances the formation of cytoprotective bioactive lipids - lipoxins, resolvins and protectins - that, in turn, suppress production of proinflammatory prostaglandins, free radicals and cytokines. The beneficial actions of albumin thus depend on its ability to mobilize PUFAs and the formation of adequate amounts of lipoxins, resolvins and protectins. For those who have hepatic and tissue deficiency of PUFAs, albumin fails to mobilize PUFAs and formation of lipoxins, resolvins and protectins will be inadequate, which may explain failure of the beneficial actions of albumin in the critically ill.

\section{Metabolism of polyunsaturated fatty acids and formation of lipoxins, resolvins and protectins}

The cell membrane is rich in PUFAs (arachidonic acid, eicosapentaenoic acid and docosahexaenoic acid) that give rise to both proinflammatory prostaglandins, thromboxanes and leukotrienes and anti-inflammatory lipoxins, resolvins and protectins [9] (see Figure 1). Balance between these mutually antagonistic compounds could determine the final outcome of the disease process $[9,10]$.

Correspondence: Undurti@hotmail.com

1 UND Life Sciences, 2020 S 360th St, \# K-202, Federal Way, WA 98003, USA ${ }^{2}$ Department of Medicine and Bio-Science Research Centre, GVP Hospital, Gayatri Vidya Parishad College of Engineering Campus, Visakhapatnam 533 048, India

\section{Albumin has anti-inflammatory actions}

Plasma concentrations of TNF $\alpha$, interleukin (IL)- 6 and macrophage inflammatory protein-2 were significantly lower and the IL-10 concentration higher in the albumin-treated hemorrhagic shock model animals [12]. Hypoalbuminemia thus results in decreased formation of IL-10 and lipoxins, resolvins and protectins that leads to increased morbidity and mortality [2]. The amount of PUFAs stored in the liver could therefore be one variable that influences formation of lipoxins, resolvins and protectins. Decreased activity of cyclooxygenases and 5lipoxygenase, 12-lipoxygenase, and 15-lipoxygenase concerned with the formation of lipoxins, resolvins and protectins is yet another variability that contributes to the controversial responses to albumin therapy reported. The albumin half-life time is shorter and its transportation rate is higher in sepsis compared with control individuals [11], which results in inadequate availability of albumin to tissues in sepsis.

\section{Tumor necrosis factor alpha induces hypoalbuminemia and polyunsaturated fatty acid deficiency}

TNF $\alpha$ administration to healthy well-nourished rabbits produced hypoalbuminemia [13] and endothelial cells 


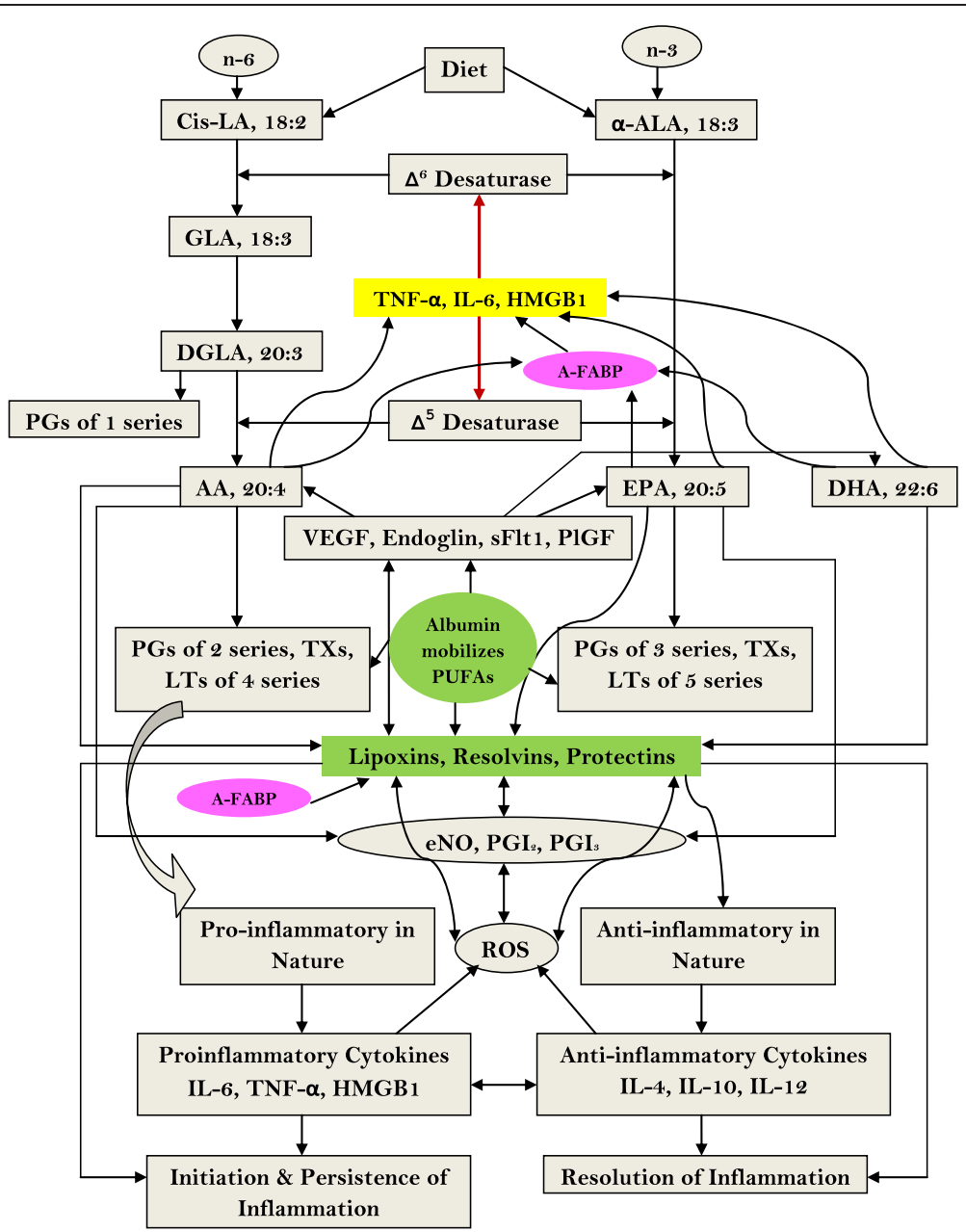

Figure 1 Metabolism of essential fatty acids and their role in inflammation and its resolution. $A A$, arachidonic acid; A-FABP, adipose-fatty acid binding protein; ALA, alpha-linolenic acid; DGLA, dihomo-gamma-linolenic acid; DHA, docosahexaenoic acid; eNO, endothelial nitric oxide; EPA, eicosapentaenoic acid; GLA, gamma-linolenic acid; HMGB1, high-mobility group box 1; IL, interleukin; LA, linolenic acid; LT, leukotriene; PG, prostaglandin; PIGF, placental growth factor; PGI, prostacyclin; PUFA, polyunsaturated fatty acid; ROS, reactive oxygen species; sFlt1, Soluble fms-like tyrosine kinase 1; TNFa, tumor necrosis factor alpha; TX, thromboxane; VEGF, vascular endothelial growth factor.

treated with TNFo showed a decrease in their PUFA content and developed essential fatty acid deficiency [14]. These results suggest that enhanced circulating levels of TNF $\alpha$ and IL-6 seen in sepsis induce hypoalbuminemia and PUFA deficiency that leads to decreased production of lipoxins, resolvins and protectins. Furthermore, both vascular endothelial growth factor and placental growth factor that are increased in sepsis can be modulated by lipoxins, resolvins and protectins [15].

\section{Conclusions}

Based on the preceding discussion, I propose that PUFAs need to be administered along with albumin to enhance formation of lipoxins, resolvins and protectins to derive the benefit of albumin therapy in the critically ill $[16,17]$.

Beneficial actions of albumin are limited by: availability of PUFAs in the liver and other tissues; formation of adequate amounts of lipoxins, resolvins and protectins from PUFAs; activity of cyclooxygenase and lipoxygenase enzymes; circulating levels of TNF $\alpha$ and other cytokines that interfere with the formation of arachidonic acid, eicosapentaenoic acid and docosahexaenoic acid and induce hypoalbuminemia; enhanced formation of reactive oxygen species that peroxidize PUFAs and thus reduce their availability; and formation of proinflammatory prostaglandins that antagonize actions of lipoxins, resolvins and protectins $[16,17]$ (see Figure 1). In view of the interactions among albumin, PUFAs, free radicals, prostaglandins, lipoxins, resolvins and protectins, nitric oxide (PUFAs and lipoxins enhance endothelial nitric oxide generation and protect endothelial cells), vascular endothelial growth factor, placental growth factor and cytokines, it is not surprising that albumin infusion alone is unlikely to be of benefit in sepsis. Hence, albumin needs 
to be given along with PUFAs and lipoxins to antagonize the actions of proinflammatory molecules and restore homeostasis [16,17].

Adipose-fatty acid binding protein (A-FABP), which induces insulin resistance and whose levels are increased in the critically ill [18], is a carrier of fatty acids and is expressed primarily in adipocytes and macrophages. AFABP regulates systemic insulin sensitivity and lipid and glucose metabolism [19]. Mice deficient in A-FABP are resistant to the development of hyperinsulinemia, hyperglycemia and insulin resistance [18].

A-FABP can be linked to the expression of Toll-like receptors, macrophage activation, synthesis and release of proinflammatory cytokines IL- 6 and TNF $\alpha$, activation of cyclooxygenase- 2 expression and eicosanoid synthesis, events that cause insulin resistance and initiation and progression of inflammation and sepsis. PUFAs and lipoxins, resolvins and protectins suppress A-FABP expression, inhibit macrophage and cyclooxygenase-2 activation, and decrease production of proinflammatory cytokines, and thus decrease insulin resistance and resolve inflammation and augment recovery from sepsis $[18,19]$. Hence, serial measurement of both proinflammatory and anti-inflammatory molecules and correlation of their levels with the progression to or recovery from (or both) sepsis and other inflammatory processes may help to predict prognosis in inflammatory conditions and eventually could lead to the development of new therapeutic strategies.

\footnotetext{
Abbreviations

A-FABP: Adipose-fatty acid binding protein; IL: Interleukin;

PUFA: Polyunsaturated fatty acid; TNFa: Tumor necrosis factor alpha.
}

\section{Competing interests}

The author declares that he has no competing interests.

\section{Acknowledgements}

UND is in receipt of a Ramalingaswami Fellowship from the Department of Biotechnology, New Delhi, India. This work is supported by grants from the Department of Biotechnology (BT/PR11627/MED/30/157/2010), the Department of Science and Technology (IR/SO/LU/03/2008/1) and the Defence Research and Development Organisation, New Delhi (TC/2519/INM-03/ 2011/CARS) under R\&D Project INM-311.

Published online: 30 March 2015

\section{References}

1. Finfer S, McEvoy S, Bellomo R, McArthur C, Myburgh J, Norton R, et al. Impact of albumin compared to saline on organ function and mortality of patients with severe sepsis. Intensive Care Med. 2011;37:86-96.

2. Caraceni P, Domenicali M, Tovoli A, Napoli L, Ricci CS, Tufoni M, et al. Clinical indications for the albumin use: still a controversial issue. Eur J Intern Med. 2013;24:721-8.

3. Intravenous fluid therapy in adults in hospital: NICE clinical guideline 174 (December 2013). http://www.nice.org.uk/guidance/cg174/resources/ guidance-intravenous-fluid-therapy-in-adults-in-hospital-pdf. Accessed 10 February 2015

4. Dellinger RP, Levy MM, Rhodes A, Annane D, Gerlach H, Opal SM, et al. Surviving sepsis campaign guidelines committee including the pediatric subgroup. Surviving sepsis campaign: international guidelines for management of severe sepsis and septic shock, 2012. Intensive Care Med. 2013;39:165-228.

5. Guidet B, Mosqueda GJ, Priol G, Aegerter P. The COASST study: costeffectiveness of albumin in severe sepsis and septic shock. J Crit Care. 2007;22:197-203

6. Patel A, Laffan MA, Waheed U, Brett SJ. Randomised trials of human albumin for adults with sepsis: systematic review and meta-analysis with trial sequential analysis of all-cause mortality. BMJ. 2014;349:g4561.

7. Dubois MJ, Orellana-Jimenez C, Melot C, De Backer D, Berre J, Leeman M, et al. Albumin administration improves organ function in critically ill hypoalbuminemic patients: a prospective, randomized, controlled, pilot study. Crit Care Med. 2006;34:2536-40.

8. Delaney AP, Dan A, McCaffrey J, Finfer S. The role of albumin as a resuscitation fluid for patients with sepsis: a systematic review and meta-analysis. Crit Care Med. 2011;39:386-91.

9. Das UN. Molecular basis of health and disease. New York: Springer; 2011.

10. Das UN. Albumin and lipid enriched albumin for the critically ill. J Assoc Physicians India. 2009;57:53-63.

11. Das UN. Albumin to globulin ratio and/or plasma albumin in predicting long-term mortality. Am J Surg. 2014;208:157-8.

12. Zhang $\mathrm{H}$, Voglis $\mathrm{S}$, Kim CH, Slutsky AS. Effects of albumin and Ringer's lactate on production of lung cytokines and hydrogen peroxide after resuscitated hemorrhage and endotoxemia in rats. Crit Care Med. 2003;31:1515-22.

13. Hennig B, Honchel R, Goldblom SE, McClaiw CJ. Tumor necrosis factormediated hypoalbuminemia in rabbits. J Nutr. 1988;119:1586-90.

14. Mayer K, Schmidt R, Muhly-Reinholz M, Bögeholz T, Gokorsch S, Grimminger $F$, et al. In vitro mimicry of essential fatty acid deficiency in human endothelial cells by TNF- $\alpha$ impact of $\omega-3$ versus $\omega-6$ fatty acids. J Lipid Res. 2002;43:944-51.

15. Cezar-de-Mello PF, Nascimento-Silva V, Villela CG, Fierro IM. Aspirin-triggered lipoxin A4 inhibition of VEGF-induced endothelial cell migration involves actin polymerization and focal adhesion assembly. Oncogene. 2006;25:122-9.

16. Das UN. HLA-DR expression, cytokines and bioactive lipids in sepsis. Arch Med Sci. 2014;10:325-35.

17. Das UN. Is sepsis a pro-resolution deficiency disorder? Med Hypoth. 2013;80:297-9

18. Huang C-L, Wu Y-W, Hsieh A-R, Hung Y-H, Chen W-J, Yang W-S. Serum adipocyte fatty acid-binding protein levels in critical illness patients are associated with insulin resistance and predict mortality. Crit Care. 2013;17:R22.

19. Das UN. Serum adipocyte fatty acid-binding protein in the critically ill. Crit Care. 2013;17:121. 